Revista Destaques Acadêmicos, Lajeado, v. 8, n. 4, 2016. ISSN 2176-3070

DOI: http://dx.doi.org/10.22410/issn.2176-3070.v8i4a2016.1212

www.univates.br/revistas

\title{
DESIGN E SIMULAÇÃO DE UM SENSOR SEM FIO PARA MONITORAMENTO REMOTO DE TRINCAS EM ESTRUTURAS METÁLICAS
}

\author{
Matheus Freitas Kuhn ${ }^{1}$, Thomas Clarke ${ }^{2}$
}

\begin{abstract}
Resumo: Tecnologias para o monitoramento de estrututras metálicas, tais como strain gages e fibra óptica, são usualmente utilizadas quando se deseja obter dados de deformação. Ambas as técnicas exigem cabeamento, produzindo diversas adversidades em suas aplicações. Assim, novas tecnologias sem fio ganham espaço, buscando monitoramento remoto e versatilidade. Sensores sem fio utilizando a tecnologia RFID (Identificação por radiofrequência) se mostram atrativos meios de contornar tais adversidades. Estes sensores são formados por uma antena e um semicondutor de identificação (RFID). O conceito básico do sensor é que este, quando colado ao material que sofre deformação, irá se deformar também, alterando sua frequência de ressonância. Dessa forma, tanto para tração quanto para compressão, teremos variadas respostas. Para construção do sensor foi utilizado o material FR-4 e para o corpo de prova uma chapa de alumínio de 16mm seguindo a norma E647 para ensaios de taxa de crescimento de trincas por fadiga. Foi realizado um estudo numérico com elementos finitos com a finalidade de validar o sensor. À medida que a trinca se propaga, os dados da frequência de ressonância são coletados e estes relacionados.
\end{abstract}

Palavras-chave: Sensor. Trinca. Passivo. RFID.

\section{INTRODUÇÃO}

Estruturas metálicas são constantemente submetidas a esforços com cargas altas e cíclicas. Estes esforços podem levar à nucleação de trincas e falha da estrutura em operação. A fim de prevenir e detectar este problema, técnicas de monitoramento de estruturas são utilizadas. Dentre as novas técnicas, se encontram os sensores com RFID (Radio Frequency Identification), por ser este um método que não exige cabeamento e possui alta probabilidade de detecção (YI et al. 2012). Buscando novas aplicações em análise de integridade estrutural e

1 Engenheiro Metalúrgico - LAMEF/Universidade Federal do Rio Grande do Sul.

2 Doutor, Professor- LAMEF/ Universidade Federal do Rio Grande do Sul. 
soluções eficientes e econômicas, se faz necessário o desenvolvimento contínuo de novas técnicas.

Recentemente, novos sensores de deformação sem fio vêm sendo estudados fazendo uso de técnicas de radiofrequência. O mecanismo base deste sensor consiste em um circuito ressonante que ao sofrer tensão/deformação sua frequência de ressonância eletromagnética também varia (THAI , 2011).

O RFID é um sistema de armazenagem de dados, tipicamente utilizado para identificação de objetos. Neste trabalho, por se tratar de um sistema passivo sua fonte de energia e troca de dados é realizado através de ondas eletromagnéticas. $\mathrm{O}$ sensor é composto de duas partes; uma antena e um Circuito Integrado (CI). A antena coleta energia da onda eletromagnética e transmite para o $\mathrm{CI}$, onde ocorre a modulação do sinal e posterior reflexão (LANTZ, 2011).

O Método dos Elementos Finitos (MEF) é um método numérico aproximado para análise de diversos fenômenos físicos que ocorrem em meios contínuos. Esses elementos são descritos por equações diferenciais e resolvidos por modelos matemáticos.

O presente trabalho tem como principal objetivo validar um sensor sem fio para monitoramento remoto de trincas em estruturas metálicas, construído através do método dos elementos finitos e posterior processamento dos dados obtidos.

\section{MATERIAIS E MÉTODOS}

Neste trabalho foi utilizado o pacote comercial de simulação numérica COMSOL Multiphysics. Este software baseado em MEF tornou possível reunir todas as ferramentas necessárias para a realização deste trabalho. $\mathrm{O}$ método de resolução do problema baseou-se em duas físicas: Solid Mechanics e Electromagnetic Waves. O sensor, antena + CI, foi projetado com material FR4, de $35 \mu \mathrm{m}$ de cobre em ambas as faces do sensor, e $1,5 \mathrm{~mm}$ de espessura. $\mathrm{O}$ tipo de antena do projeto é chamada de microstrip slotted patch antenna e tornou-se atrativo pois a camada de cobre inferior, ground, reduz os efeitos deletérios aos parâmetros desejados (CHO, 2010). Utilizou-se um CI com impedância de 21,9 - 253i ohms. O corpo de prova foi projetado em alumínino com espessura de $16 \mathrm{~mm}$ e segue a norma E647 para ensaios de taxa de crescimento de trincas por fadiga. As dimensões do sensor e corpo de prova são ilustradas na Figura 1. 
Figura 1 - Dimensões corpo de prova e sensor em milímetros

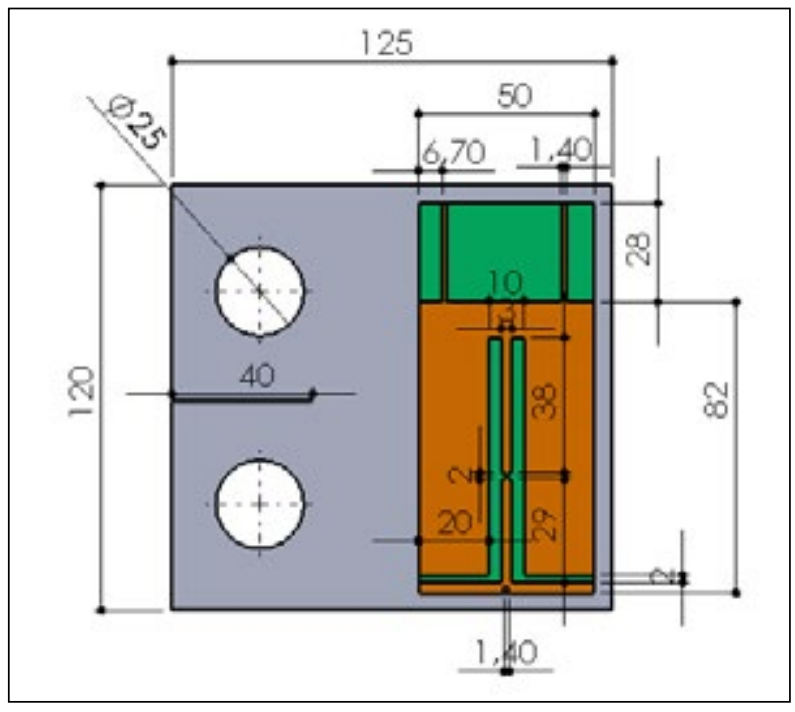

Fonte: dos autores.

Previamente à simulação da propagação da trinca, foi realizada uma simulação com deslocamentos uniaxiais com o propósito de verificar o comportamento da frequência de ressonância ao variar com a aplicação de deslocamentos, uma vez que a frequência de ressonância segue a equação 1 , abaixo:

Equação 1 - Frequência de ressonância de uma microstrip slotted patch antenna.

$f_{0}=\frac{c}{8\left(L+L^{\prime}\right) \sqrt{\varepsilon_{r e f f}}}$

Onde $L$ é o comprimento físico da antena, $L^{\prime}$ é uma extensão do comprimento devido ao efeito de borda, $c$ é a velocidade da luz no vácuo e $\varepsilon_{\text {reff }}$ é a constante dielétrica efetiva. Ao sofrer os deslocamentos, o valor do comprimento físico da antena deve aumentar e assim reduzir a frequência de ressonância.

Assim se inicia um estudo paramétrico em uma placa de alumínio variando de $0 \mu \mathrm{m}$ até $1500 \mu \mathrm{m}$ e deslocamentos com 12 intervalos entre eles. A análise seguiu dois passos; um estacionário e outro no domínio da frequência. Devido a problemas de convergência dos resultados, escolheu-se realizar estes dois passos em arquivos separados, ou seja, primeiramente foi realizado um estudo estacionário no qual se aplicou os deslocamentos através da física Solid Mechanics. Após a aplicação deste, foi utilizada a função Remesh Deformed Configuration, a qual permite que a malha deformada possa se tornar um objeto 
e este ser exportado. A Figura 2 apresenta a posição do sensor e o sentido dos deslocamentos aplicados.

Figura 2 - Sentido dos deslocamentos

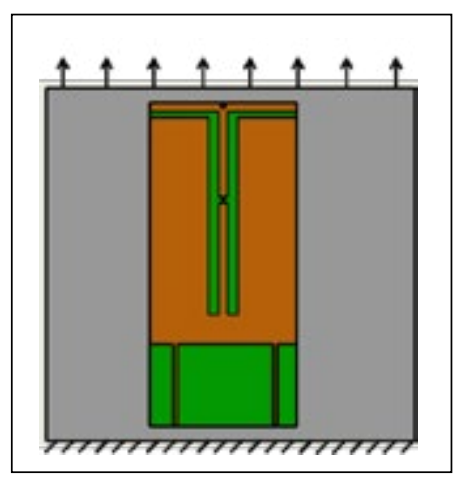

Fonte: dos autores.

Em sequência, inicia-se a análise no domínio da frequência. A análise da frequência de ressonância do sensor foi realizada na física Electromagnetic Waves. Nesta física, a região onde o sensor sofrerá a excitação se chama Lumped Port, onde foi aplicada uma impedância de 21.9 -253i ohms. É importante ressaltar a necessidade de se obter uma antena com valor de impedância próximo à $21.9+$ $253 \mathrm{iohms}$, para assim obter a máxima transferência de potência em um circuito RLC (DOBKIN, 2013). As superfícies do cobre são simuladas com Perfect Electric Conductor a fim de simplificar o modelo, pois assim a onda incidente não terá perda e irá refletir integralmente para análise. Os resultados plotados são gráficos do parâmetro S11 em função da frequência para cada deslocamento aplicado. O menor valor dde S11 possui uma frequência correspondente que pode ser chamada de frequência de ressonância.

Seguindo a mesma metodologia, foi realizado um estudo paramétrico referente a propagação da trinca, onde se observou o crescimento da trinca até o valor de $15 \mathrm{~mm}$, com intervalos de $1 \mathrm{~mm}$. A simulação da trinca consistiu em propagar um vazio de $0,1 \mathrm{~mm}$ de altura no corpo de prova e no sensor e na etapa do carregamento do corpo de prova foi aplicada uma força de $5000 \mathrm{~N}$ ao mesmo tempo que a outra região foi engastada, vide Figura 3. 
Figura 3 - Carregamento do corpo de prova

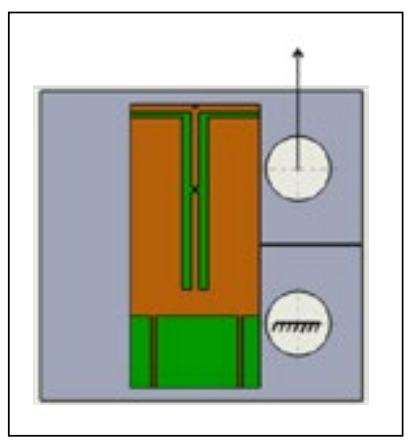

Fonte: dos autores.

Por fim, aplicou-se a mesma rotina da simulação anterior gerando resultados que se encontram nos gráficos do parâmetro S11 em função da frequência para cada comprimento da trinca.

\section{RESULTADOS E DISCUSSÃO}

Os resultados obtidos para o sensor consistem em uma frequência de ressonância de $906,2 \mathrm{MHz}$ adequada à banda de ultra-alta frequência, 860$990 \mathrm{MHz}$, e uma impedância com valor de 15,62 +253,4i ohms, como ilustrado nas Figuras 4 e 5 respectivamente.

Deve-se observar que este sensor foi projetado e posicionado sobre o corpo de prova, uma vez que estruturas metálicas causam alterações nos parâmetros desejados do sensor (BYUNGGIL, 2010; YE, 2014). 
Figura 4 - Frequência de ressonância

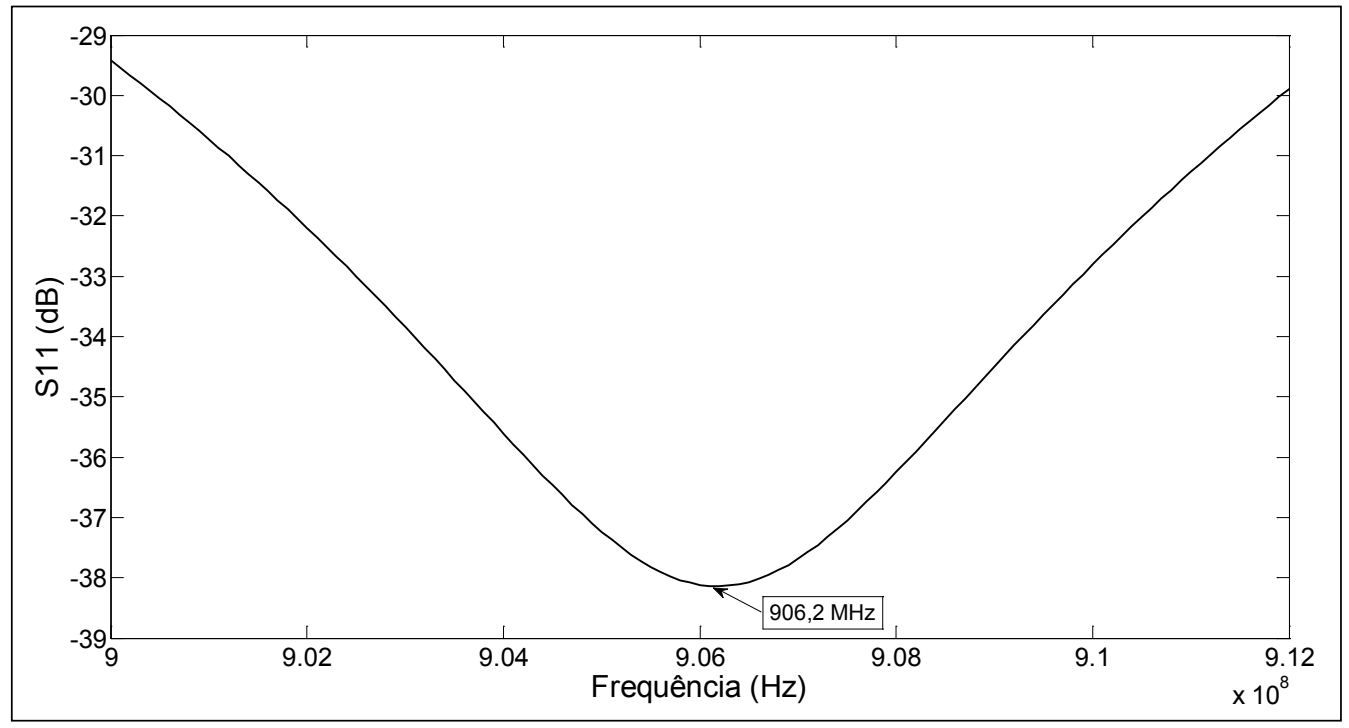

Fonte: dos autores.

Figura 5 - Impedância do sensor

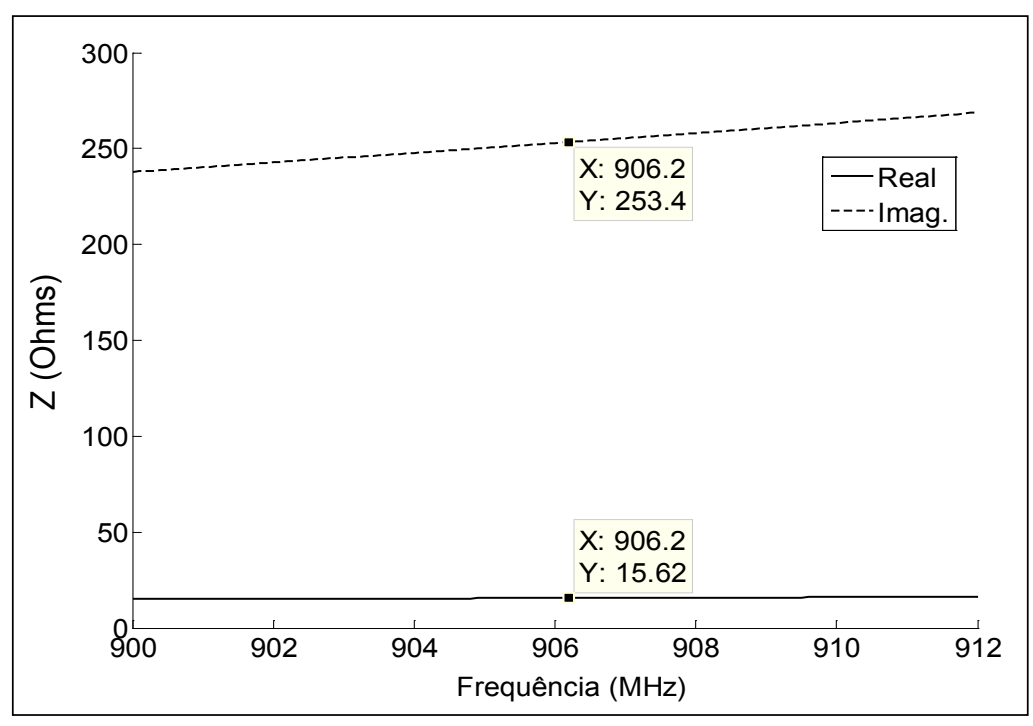

Fonte: dos autores.

O diagrama de radiação, vide Figura 6, apresenta a direção na qual o sensor irá responder com eficiência superior; direção perpendicular à face superior do sensor. 
Figura 6 - Diagrama de radiação

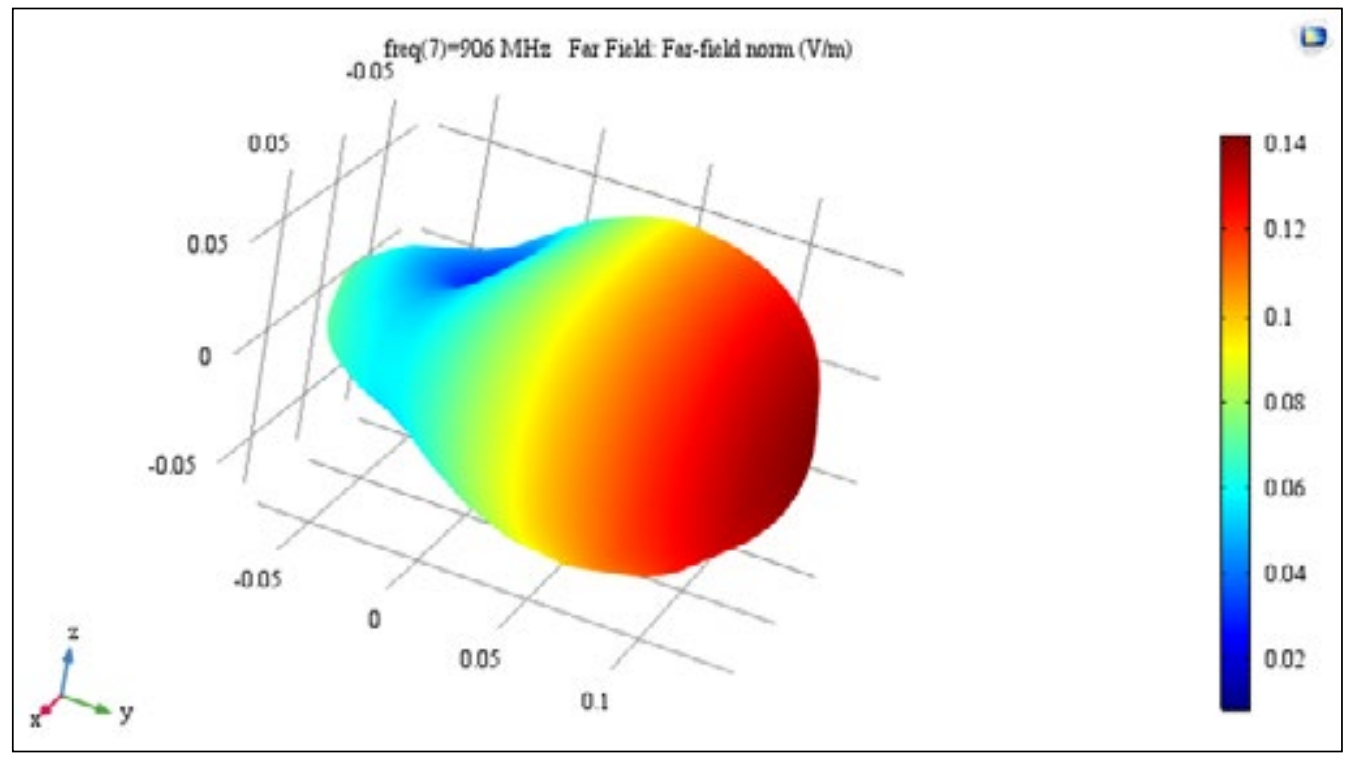

Fonte: dos autores.

Na simulação do sensor a partir dos deslocamentos aplicados se obteve os resultados ilustrados na Figura 7. É notável que na ocorrência do aumento no deslocamento, há redução na frequência de ressonância, acordando com a equação 1. Os valores da frequência de ressonância foram coletados a partir do menor valor de S11.

O resultado mostrado na Figura 8 está de acordo com o esperado. Os pontos representantes das frequências de ressonância estão fortemente relacionados à tendência linear entre a frequência de ressonância e $o$ deslocamento, este tipo de tendência também foi encontrado nos trabalhos de Lantz (2011) e Yi (2012). 
Figura 7 - Curvas de S11 em relação à frequência para deslocamento aplicados

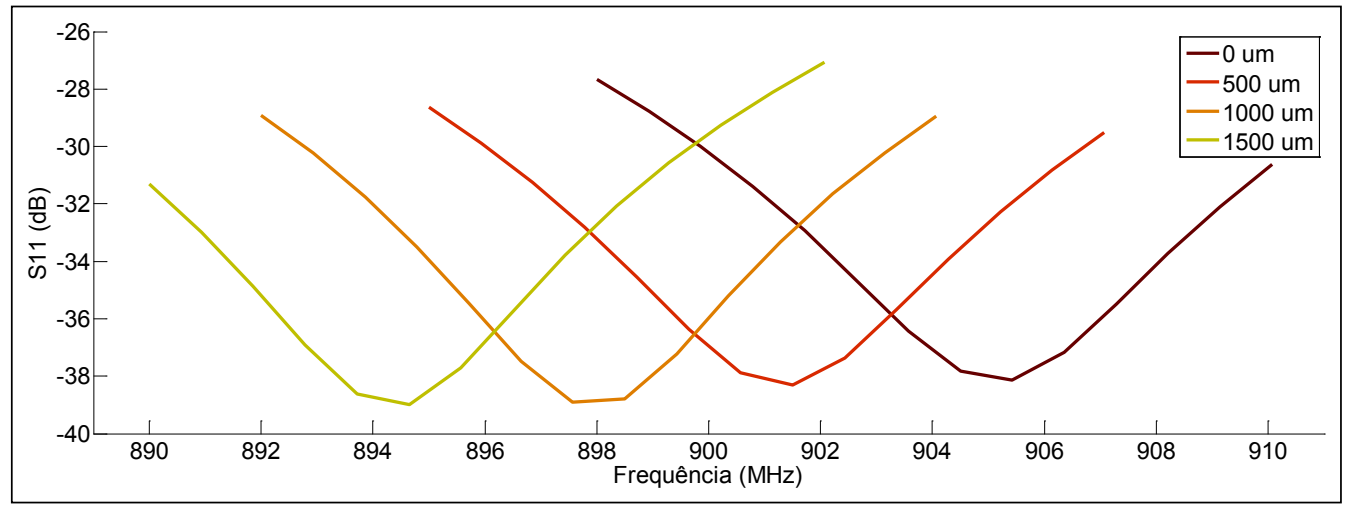

Fonte: dos autores.

Figura 8 - Relação entre a frequência de ressonância e o deslocamento.

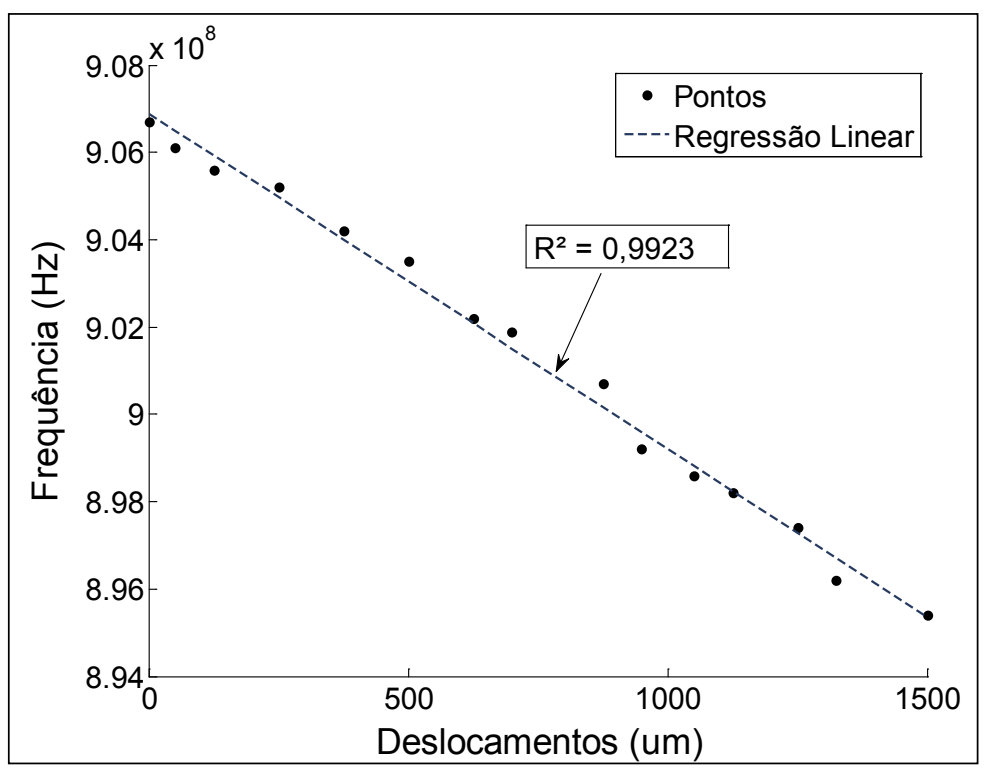

Fonte: dos autores.

Para a simulação do efeito da propagação da trinca no material, foram obtidos os resultados mostrados na Figura 9. O comportamento de forma semelhante à simulação dos deslocamentos foi obtido, e observada uma tendência linear representativa. Resultados semelhantes foram encontrados na literatura (DESHMUKH, 2010; YI, 2012). Devido ao estado de tensão ser bem diferente na propagação da trinca em um corpo de prova $\mathrm{CT}$ em relação aos 
deslocamentos uniaxiais, e a modificação da antena devido ao vazio gerado pela trinca, o valor para a correlação linear obtido foi considerado adequado.

$\mathrm{Na}$ Figura 10, regiões trativas possuem o valor positivo e regiões compressivas o valor negativo da escala. Estas deformações levaram a uma não linearidade da frequência de ressonância em função do comprimento da trinca, uma vez que há diferentes regiões de tração e de compressão sobre o sensor.

Figura 9 - Frequência de ressonância x comprimento da trinca

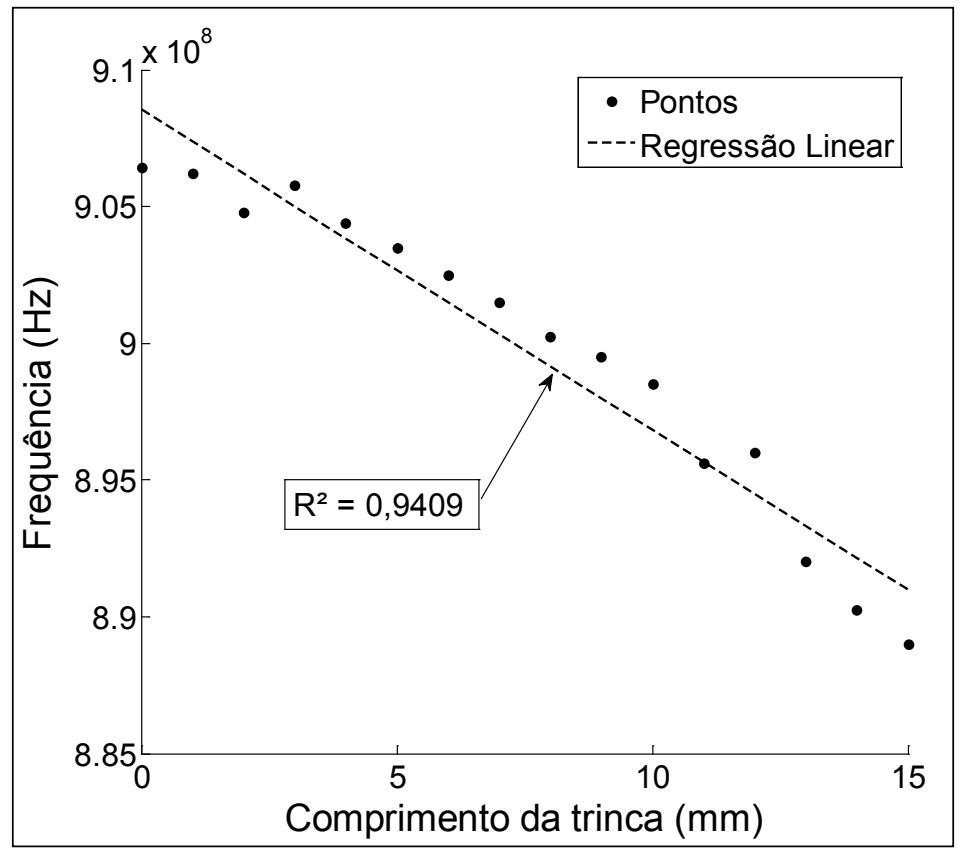

Fonte: dos autores. 
Figura 10 - Regiões trativas e compressivas no sensor

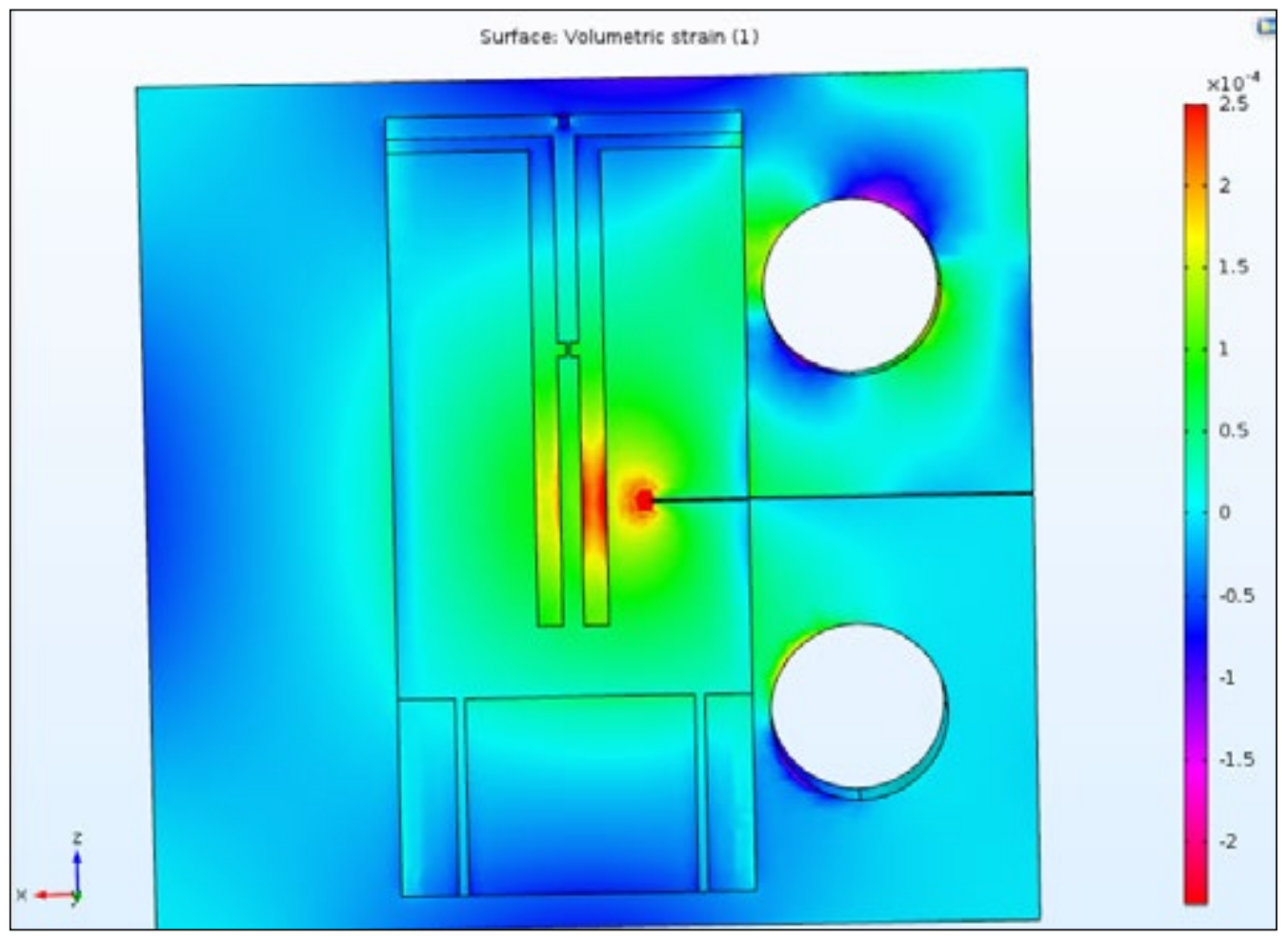

Fonte: dos autores.

\section{CONCLUSÃO}

A simulação do sensor mostrou-se capaz de detectar pequenos deslocamentos, em torno de $50 \mu \mathrm{m}$, e a propagação de defeitos, em intervalos de $1 \mathrm{~mm}$, devido a forte tendência linear tanto na aplicação dos deslocamentos quanto na propagação do defeito. Assim, devido os resultados apresentados através da simulação e a crescente necessidade de desenvolvimento de aplicações de monitoramento remoto, o sensor se mostrou solução viável, acessível e consideravelmente versátil.

\section{REFERÊNCIAS}

BYUNGGIL, Yu. RFID Tag Antennas Mountable on Metallic Platforms. Radio Frequency Identification Fundamentals and Applications Design Methods and Solutions, USA, p. 165-179, 2010. 
CHO, G.H. Design of an embedded-feed type microstrip path antenna for UHF radio frequency identificantion tag on metallic objects, IET Microwaves, Antennas \& Propagation, San Diego, Vol.4, Iss. 9, p.1232-1239, set. 2010.

DESHMUKH, Srikar. Unpowered antenna sensor for crack detection and measurement. Proc. SPIE7647, Sensors and Smart Structures Technologies for Civil, Mechanical, and Aerospace Systems 2010, San Diego, Vol 7647,p 1203-1212, abr.2010.

DOBKIN, Daniel M. The RF in RFID. Tag Antennas. 2. ed. UK, Elssevier, 2013, p.306309.

LANTZ, Gabriel. Crack detection using a passive wireless strain sensor, Georgia Institute of Technology, Atlanta, 2011.

THAI, Trang T. A Newly Developed Radio Frequency Wireless Passive Highly Sensitive Strain Transducer, Sensors, France, p. 211-214, out. 2011.

YE, Qi. Design of Robust UHF RFID Tag Antenna for Free-space and Metal Surface, Progress In Electromagnetics Research Symposium Proceedings, China, p. 22082211, Aug. 2014.

Yi, X., Fang, C., Cooper, J., Cho, C., Vyas, R., Wang, Y., Leon, R., and Tentzeris, M. ) Strain Sensing through a Passive Wireless Sensor Array. 20th Analysis and Computation Specialty Conference. Chicago p. 117-126,2012.

YI, Xiaohua. Wireless Strain and Crack Sensing Using a Folded Patch Antenna, $\mathbf{6}^{\text {th }}$ European Conference on Antennas and Propagation (EUCAP), Prague, p.1678-1681, 2012. 